\title{
561.
}

\section{ON THE GEOMETRICAL REPRESENTATION OF CAUCHY'S THEOREMS OF ROOT-LIMITATION.}

[From the Transactions of the Cambridge Philosophical Society, vol. xII. Part II. (1877), pp. 395-413. Read February 16, 1874.]

There is contained in Cauchy's Memoir "Calcul des Indices des Fonctions," Journ. de l'École Polytech. t. xv. (1837) a general theorem, which, though including a well-known theorem in regard to the imaginary roots of a numerical equation, seems itself to have been almost lost sight of. In the general theorem (say Cauchy's two-curve theorem) we have in a plane two curves $P=0, Q=0$, and the real intersections of these two curves, or say the "roots," are divided into two sets according as the Jacobian

$$
d_{x} P \cdot d_{y} Q-d_{x} Q \cdot d_{y} P
$$

is positive or negative, say these are the Jacobian-positive and the Jacobian-negative roots: and the question is to determine for the roots within a given contour or circuit, the difference of the numbers of the roots belonging to the two sets respectively.

In the particular theorem (say Cauchy's rhizic theorem) $P$ and $Q$ are the real part and the coefficient of $i$ in the imaginary part of a function of $x+i y$ with, in general, imaginary coefficients (or, what is the same thing, we have $P+i Q=f(x+i y)+i \phi(x+i y)$, where $f, \phi$ are real functions of $x+i y)$ : the roots of necessity are of the same set: and the question is to determine the number of roots within a given circuit.

In each case the required number is theoretically given by the same rule, viz. considering the fraction $\frac{P}{Q}$, it is the excess of the number of times that the fraction changes from + to - over the number of times that it changes from - to + , as the point $(x, y)$ travels round the circuit, attending only to the changes which take place on a passage through a point for which $P$ is $=0$. 
In the case where the circuit is a polygon, and most easily when it is a rectangle the sides of which are parallel to the two axes respectively, the excess in question can be actually determined by means of an application of Sturm's theorem successively to each side of the polygon, or rectangle.

In the present memoir I reproduce the whole theory, presenting it under a completely geometrical form, viz. I establish between the two sets of roots the distinction of right- and left-handed: and (availing myself of a notion due to Prof. Sylvester*) I give a geometrical form to the theoretic rule, making it depend on the "intercalation" of the intersections of the two curves with the circuit: I also complete the Sturmian process in regard to the sides of the rectangle: the memoir contains further researches in regard to the curves in the case of the particular theorem, or say as to the rhizic curves $P=0, Q=0$.

\section{The General Theory. Articles Nos. 1 to 19.}

1. Consider in a plane two curves $P=0, Q=0(P$ and $Q$ each a rational and integral function of $x, y$ ), which to fix the ideas I call the red curve and the blue curve respectively $\dagger$ : the curve $P=0$ divides the plane into two sets of regions, say a positive set for each of which $P$ is positive, and a negative set for each of which $P$ is negative: it is of course immaterial which set is positive and which negative, since writing $-P$ for $P$ the two sets would be interchanged: but taking $P$ to be given, the two sets are distinguished as above. And we may imagine the negative regions to be coloured red, the positive ones being left uncoloured, or say they are white. Similarly the curve $Q=0$ divides the plane into two sets of regions, the negative regions being coloured blue, and the positive ones being left uncoloured, or say they are white. Taking account of the twofold division, and considering the coincidence of red and blue as producing black, there will be four sets of regions, which for convenience may be spoken of as sable, gules, argent, azure: viz, in the figures we have

$$
\begin{aligned}
& \overbrace{-}^{P Q} \text { sable, shown by cross lines, } \\
& -+ \text { gules, " " " vertical lines, } \\
& ++ \text { argent, left white, } \\
& +- \text { azure, shown by horizontal lines, }
\end{aligned}
$$

sable and argent $(--$ and ++$)$ being thus positive colours, and gules and azure $(-+$ and +-$)$ negative colours. See figures [pp. 32, 38] towards end of Memoir.

* See his memoir, "A theory of the Syzygetic relations, \&c." Phil. Trans., 1853. The Sturmian process is by Sturm and Cauchy applied to two independent functions $\phi x, f x$ of a variable $x$; but the notion of an intercalation as applied to the order of succession of the roots of the equations $\phi(x)=0, f(x)=0$ is due to Sylvester, and it was he who showed that what the Sturmian process determined was in fact the intercalation of these roots: but, not being concerned with circuits, he was not led to consider the intercalation of a circuit.

+ It is assumed throughout that the two curves have no points (or at least no real points) of multiple intersection; i.e. they nowhere touch each other, and neither curve passes through a multiple point of the other curve. 
2. Consider any point of intersection of the two curves. There will be about this point four regions, sable and argent being opposite to each other, as also gules and azure; whence selecting an order

$$
\text { sable, gules, argent, azure; }
$$

if to have the colours in this order we have to go about the point, or root, righthandedly, the root is right-handed: but if left-handedly, then the root is left-handed: or, what is more convenient, going always right-handedly, then, if the order of the colours is

$$
\text { sable, gules, argent, azure, }
$$

the root is right-handed: but if the order is

$$
\text { sable, azure argent, gules, }
$$

the root is left-handed.

3. The distinction of right- and left-handed corresponds to the sign of the Jacobian

$$
\frac{d(P, Q)}{d(x, y)}\left(=d_{x} P \cdot d_{y} Q-d_{x} Q \cdot d_{y} P\right)
$$

we may (reversing if necessary the original sign of one of the functions) assume that for a right-handed root the Jacobian is positive, for a left-handed one, negative.

4. I consider a trajectory which may be either an unclosed curve not cutting itself, or else a circuit, viz. this is a closed curve not cutting itself. A circuit is considered as described right-handedly: an unclosed trajectory is considered as described according to a currency always determinate pro hâc vice: viz. one extremity is selected as the beginning and the other as the end of the trajectory: but the currency may if necessary or convenient be reversed: thus if an unclosed trajectory forms part of a circuit the currency is thereby determined: but the same unclosed trajectory may form part of two opposite circuits, and as such may have to be taken with opposite currencies. It is assumed that a trajectory does not pass through any intersection of the $P$ and $Q$ curves.

5. A trajectory has its $P$ - and $Q$-sequence, viz. considering in order its intersections with the two curves, we write down a $P$ for each intersection with the red curve and a $Q$ for each intersection with the blue curve, thus obtaining an intermingled series of $P$ 's and $Q$ 's, which is the sequence in question. In the case of a circuit, the sequence is considered as a circuit, viz. the first and last terms are considered as contiguous, and it is immaterial at what point the sequence commences. The sequence will of course vanish if the trajectory does not meet either of the curves.

6. A $P$ - and $Q$-sequence gives rise to an "intercalation," viz. if in the sequence there occur together any even number of the same letter, these are omitted (whence also any odd number of the same letter is reduced to the letter taken once): and if by reason of an omission there again occur an even number of the same letter, these 
are omitted: and so on. The intercalation contains therefore only the letters $P$ and $Q$ alternately: viz. in the case of an unclosed trajectory the intercalation may contain an even number of letters, beginning with the one and ending with the other letter, and so containing the same number of each letter-or it may contain an odd number of letters, beginning and ending with the same letter, and so containing one more of this than of the other letter; say the intercalation is $P Q$ or $Q P$, or else $P Q P$ or $Q P Q$. The intercalation may vanish altogether; thus if the sequence were $Q P P Q$, this would be the case.

7. In the case of a circuit the intercalation cannot begin and end with the same letter, for these, as contiguous letters, would be omitted; and since any letter thereof may be regarded as the commencement it is $P Q$ or $Q P$ indifferently. A little consideration will show that the whole number of letters must be evenly even, or, what is the same thing, the number of each letter must be even. Thus imagine the circuit beginning in sable, and let the intercalation begin with $P Q$; viz. $P$ we pass from sable to azure, and $Q$ we pass from azure to argent: in order to get back into sable we must either return the same way ( $Q$ argent to azure, $P$ azure to sable), but then the sequence is $P Q Q P$, and the intercalation vanishes: here the number of letters is 0 , an evenly even number: or else we must complete the cycle of colours $P$ argent to gules, $Q$ gules to sable: and the sequence and therefore also the intercalation then is $P Q P Q$, where the number of letters is 4 , an evenly even number.

8. In the case of any trajectory whatever, the half number of letters in the intercalation is termed the "index," viz. this is either an integer or an integer $+\frac{1}{2}$. But in the case of a circuit the index is an even integer, and the half-index is therefore an integer. The index may of course be $=0$.

9. But we require a further distinction: instead of a $P$ - and $Q$-sequence we have to consider a $\pm P$ - and $Q$-sequence. To explain this, observe that a passage over the red curve may be from a negative to a positive colour (azure to sable or gules to argent), this is $+P$, or from a positive to a negative colour (sable to azure or argent to gules), this is $-P$. And so the passage over the blue curve may be from a negative to a positive colour (gules to sable or azure to argent), this is $+Q$, or else from a positive to a negative colour (sable to gules or argent to azure), this is $-Q$. The sequence will contain the $P$ and $Q$ intermingled in any manner, but the signs will always be +- alternately; for $+(P$ or $Q)$, denoting the passage into a positive colour, must always be immediately succeeded by $-(P$ or $Q)$, denoting the passage into a negative colour. Whence, knowing the sequence independently of the signs, we have only to prefix to the first letter the sign + or - as the case may be, and the sequence is then completely determined.

10. Passing to a \pm intercalation, observe that in omitting any even number of $P^{\prime}$ 's or $Q$ 's, the omitted signs are always $+-+-\&$ c. or else $-+-+\&$ c., viz. the omitted signs begin with one sign and end with the opposite sign. Hence the signs being in the first instance alternate, they will after any omission remain alternate: and the letters being also alternate, the intercalation can contain only $+P$ and $-Q$ 
or else $-P$ and $+Q$. Hence in the case of a circuit the intercalation is either $(+P-Q)$, say this is a positive circuit, or else $(-P+Q)$, say this is a negative circuit. There is of course the neutral circuit $(P Q)_{0}$ for which the intercalation vanishes.

11. Consider a circuit not containing within it any root; as a simple example let the circuit lie wholly in one colour, or wholly in two adjacent colours, say sable and gules: in the former case the sequence, and therefore also the intercalation, vanishes: in the latter case the sequence is $+Q-Q$, and therefore the intercalation vanishes: viz. in either case the intercalation is $(P Q)_{0}$.

12. Consider next a circuit containing within it one right-handed root; for instance let the circuit lie wholly in the four regions adjacent to this root, cutting the two curves each twice; the sequence and therefore also the intercalation is $+P-Q+P-Q$; viz. this is a positive circuit $(+P-Q)_{1}$, where the subscript number is the half-index, or half of the number of $P$ 's or of $Q$ 's. Similarly if a circuit contains within it one left-handed root, for instance if the circuit lies wholly in the four regions adjacent to this root, cutting the two curves each twice, the sequence and therefore also the intercalation is $-P+Q-P+Q$, viz. this is a negative circuit $(-P+Q)_{1}$ : and the consideration of a few more particular cases leads easily to the general and fundamental theorem :

13. A circuit is positive $(+P-Q)_{\delta}$ or negative $(-P+Q)_{\delta}$ according as it contains within it more right-handed or more left-handed roots; and in either case the half-index $\delta$ is equal to the excess of the number of one over that of the other set of roots. If the circuit is neutral $(P Q)_{0}$, then there are within it as many left-handed as righthanded roots.

14. The proof depends on a composition of circuits, but for this some preliminary considerations are necessary.

Imagine two unclosed trajectories forming a circuit, and write down in order the intercalation of each. The whole number of letters must be even: viz. the numbers for the two intercalations respectively must be both even or both odd. I say that if the terminal letter of the first intercalation and the initial letter of the second intercalation are different, then also the initial letter of the first intercalation and the terminal letter of the second intercalation will be different: if the same, then the same. In fact, the intercalations may be each $P Q$ or each $Q P$, or one $P Q$ and the other $Q P$ : or each $P Q P$, or each $Q P Q$, or one $P Q P$ and the other $Q P Q$. Supposing the letters in question are different, then the intercalations may be termed similar; but if the same, then the intercalations may be termed contrary.

15. In the first case, that is when the intercalations are similar, the two together form the intercalation of the circuit; the sum of their numbers of letters (that is, twice the sum of their indices) will be evenly even, and the half of this, or the sum of the indices, will be the index of the circuit; each intercalation will be $(+P-Q)$ or else each will be $(-P+Q)$; and the circuit will be $(+P-Q)$ or $(-P+Q)$ accordingly.

c. IX. 
In the second case, that is, when the intercalations are contrary, they counteract each other in forming the intercalation of the circuit: it is the difference of the numbers of letters, or twice the difference of the indices, which is evenly even, and the half of this, or the difference of the indices, which is the index of the circuit: one intercalation is $(+P-Q)$, and the other is $(-P+Q)$ : and the circuit will agree with that which has the larger index.

In particular if the circuit consist of a single unclosed trajectory, taken forwards and backwards; then the trajectory taken one way is $(+P-Q)$, taken the other way it is $(-P+Q)$; the number of terms is of course equal, and the circuit is $(P Q)_{0}$.

16. Consider now two circuits $A B C A$ and $A C D A$, having a common portion $C A$, or, more accurately, the common portions $A C$ and $C A$ : write down in order the intercalations of

$$
A B C, C A, A C, C D A \text { : }
$$

the two mean terms destroy each other, and we can hence deduce the intercalation of the entire circuit $A B C D A$.

Suppose first, that $A B C$ and $C D A$ are similar; then if $C A$ is similar to $A B C$ it is also similar to $C D A$, that is, $A C$ is contrary to $C D A$ : and so if $C A$ is contrary to $A B C$, then $A C$ is similar to $C D A$.

To fix the ideas suppose $C A$ similar to $A B C$, but $A C$ contrary to $C D A$, then $A B C A$ is similar to $C A$; but $A C D A$ will be similar or contrary to $A C$, that is, contrary or similar to $C A$, that is, to $A B C A$, according as index of $A C>$ or $<$ index of $C D A$.

Suppose Ind. $A C<$ Ind. $C D A$, then $A C D A$ is similar to $A B C A$.

Now

and thence

$$
\begin{aligned}
& \text { Ind. } A B C D A=\text { Ind. } A B C+\text { Ind. } C D A \text {, } \\
& \text { Ind. } A B C A=\text { Ind. } A B C+\text { Ind. } A C \text {, } \\
& \text { Ind. } A C D A=\text { Ind. } C D A \text { - Ind. } A C \text {, }
\end{aligned}
$$

$$
\text { Ind. } A B C D A=\text { Ind. } A B C A+\text { Ind. } A C D A \text {, }
$$

the whole circuit being in this case similar to each of the component ones.

But if Ind. $A C>$ Ind. $C D A$, then $A C D A$ is contrary to $A B C A$.

And

and thence

Ind. $A B C D A=$ Ind. $A B C+$ Ind. $C D A$,

$$
\begin{aligned}
& \text { Ind. } A B C A=\text { Ind. } A B C+\text { Ind. } C A \text {, } \\
& \text { Ind. } A C D A=- \text { Ind. } C D A+\text { Ind. } A C,
\end{aligned}
$$

Ind. $A B C D A=$ Ind. $A B C A-$ Ind. $A C D A$;

and the investigation is like hereto if $C A$ is contrary to $A B C$ but $A C$ similar to $C D A$.

17. Secondly, if $A B C$ and $C D A$ are contrary, then if $C A$ is similar to $A B C$ it is contrary to $C D A$, that is, $A C$ is similar to $C D A$; and so if $C A$ is contrary to $A B C$ it is similar to $C D A$, that is, $A C$ is contrary to $C D A$. 
Suppose $C A$ similar to $A B C$, and $A C$ similar to $C D A$; then $A B C A$ is also similar to $A B C$, and $A C D A$ similar to $C D A$; viz. $A B C, C A$ and $A B C A$ are similar to each other, and contrary to $A C, C D A, A C D A$ which are also similar to each other.
Also
Ind. $A B C D A=$ Ind. $A B C \sim$ Ind. $C D A$,
Ind. $A B C A=$ Ind. $A B C+$ Ind. $C A$,
Ind. $A C D A=$ Ind. $C D A+$ Ind. $A C$,

and thence

Ind. $A B C D A=$ Ind. $A B C A \sim$ Ind. $A C D A$,

and the investigation is like hereto if $C A$ is contrary to $A B C$ and $A C$ contrary to $C D A$.

18. It thus appears that in every case

$$
\text { Ind. } \begin{aligned}
A B C D A & =\text { Ind. } A B C A+\text { Ind. } A C D A, \\
\text { or } & =\text { Ind. } A B C A \sim \text { Ind. } A C D A,
\end{aligned}
$$

according as the component circuits are similar or contrary, and in the latter case the entire circuit is similar to that which has the largest index.

Moreover, any circuit whatever can be broken up into two smaller circuits, and these again continually into smaller circuits until we arrive at the before-mentioned elementary circuits, and the theorem as to the number of roots within a circuit is true as regards these elementary circuits; wherefore the theorem is true as regards any circuit whatever.

19. In the case where a trajectory is a finite right line, $y$ is a given linear function of $x$, or the coordinates $x, y$ can if we please be expressed as linear functions of a parameter $u$, so that as the describing point passes along the line, $u$ varies between given limits, say from $u=0$ to $u=1$. The functions $P, Q$ thus become given rational and integral functions of a single variable $u$ (or it may be $x$ or $y$ ), and the question of the $P$ - and $Q$-sequence and intercalation relates merely to the order of succession of the roots of the equations $P=0, Q=0$, where $P$ and $Q$ denote functions of a single variable as above. To fix the ideas, let the trajectory be a line parallel to the axis of $x$; and in this case taking $x$ as the parameter, and supposing that $y_{0}$ is the given value of $y, P$ and $Q$ are the functions of $x$ obtained by writing $y_{0}$ for $y$ in the original expressions of these functions. Of course the theory will be precisely the same for a line parallel to the axis of $y$ : and by combining two lines parallel to each axis we have the case of a rectangular circuit. We require, for each side of the rectangle considered according to its proper currency, the intercalation $P Q, Q P, P Q P$ or $Q P Q$ as the case may be, and also the sign + or - of the initial letter of the first intercalation; for then writing down the intercalations in order, with the signs for the several letters, + and - alternately (the first sign being + or - as the case may be), we have or deduce the intercalation of the circuit, and thus obtain the value of the difference of the numbers of the included right- and left-handed roots. We thus see how the whole theory depends on the case where the trajectory is a right line. 
Intercalation-theory for a right line. Articles Nos. 20 to 31 .

20. Considering then the case where the trajectory is a line parallel to the axis of $x, P$ and $Q$ will denote given rational functions of $x$; the curves $P=0, Q=0$ being of course each of them a set of right lines parallel to the axis of $y$ : the regions will be bands each of them included between two such lines; and colouring them as explained in the general case, the colours will be as before, sable, gules, argent, azure, each region having in the neighbourhood of the trajectory (what we are alone concerned with) the same colour that it had in the original case where $P$ and $Q$ were functions of $(x, y)$. We may regard the trajectory as described according to the currency $x=-\infty$ to $x=+\infty$ : we have in regard to the trajectory a $P$ - and $Q$-sequence and intercalation, a $\pm P$ - and $Q$-sequence, \&c., as in the original case. The intercalation may be as before $P Q, Q P, P Q P$ or $Q P Q$, and in each of these cases it may be positive, that is, $(+P-Q)$, or else negative, that is, $(-P+Q)$.

21. The question of sign may in the present case be disposed of without difficulty. For the initial point of the trajectory, we know the signs of $P, Q$, that is, the colour of the region: suppose for example that we have $P=-, Q=+$, or that the region is gules: then if the intercalation begin with $P$, this means that we either first pass a red line, or before doing so we pass an even number of blue lines: but in the last case the colours are sable, gules, sable, gules, ... always ending in gules; and the passage over the red line is gules to argent, viz. this is $+P$; and so in general the initial $P$ or $Q$ of the intercalation has the sign opposite to that of the $P$ or $Q$ belonging to the commencement of the trajectory.

22. For the solution of the problem we connect with $P, Q$ a set of functions $R, S, T$, \&c.: the intercalation is in fact given by means of the gain or loss of changes of sign in these functions on substituting therein the initial and final values of the variable $x$. It is convenient to consider the functions as arranged in a column

$$
\begin{aligned}
& P \\
& Q \\
& R \\
& S \\
& \vdots
\end{aligned}
$$

say this is the column $P Q R S \ldots$, and to connect therewith a signaletic bicolumn: viz. the left-hand column is here the series of signs of these functions for the initial value of $x$, and the right-hand column is the series of signs for the terminal value of $x$ : the bicolumn thus consisting of as many rows each of two signs, as there are functions. But such a bicolumn may be considered apart from any series of functions, as a set of rows each of two signs taken at pleasure.

We say that the "gain" of a bicolumn is

$=-($ No. of changes of sign in left-hand column $)+($ No. in right-hand ditto), the gain being of course positive or negative; and a negative gain being regarded as a loss. Also if a positive gain be converted into an equal negative gain or vice versâ, we may speak of the gain as reversed. 
23. A bicolumn may be divided in any manner into parts, taking always the last row of any part as being also the first row of the next succeeding part. This being so, the gain of the whole bicolumn is equal to the sum of the gains of its parts.

In a bicolumn of two rows, if we reverse either row (that is, write therein - for + and + for -$)$, we reverse the gain: and hence dividing a bicolumn into bicolumns each of two rows, viz. first and second rows, second and third rows, and so on, it at once appears that if we reverse alternate rows (viz. either the first, third, fifth, \&c., rows, or the second, fourth, sixth, \&c., rows) we reverse the gain. It of course follows that reversing all the rows, we leave the gain unaltered.

24. If to any bicolumn we prefix at the top thereof the second row reversed, we either leave the gain unaltered or we alter it by \pm 1 . In fact, as regards either column, if this originally begin with a change, the process introduces no change therein; but if it begins with a continuation, then the process introduces a change. Hence if the columns begin each with a change or each with a continuation, the gain is unaltered: but if one begins with a change, and the other with a continuation, then the gain is altered by \pm 1 ; viz. the left-hand column beginning with a continuation, the gain is altered by -1 : and the right-hand column beginning with a continuation, the gain is altered by +1 .

The column PQRST... is taken to satisfy the following conditions: two consecutive terms never vanish together (that is, for the same value of the variable): if for a given value of the variable, any term vanishes, the preceding and succeeding terms have then opposite signs; the last term, say $V$, is of constant sign.

25. Considering $P, Q$ as given functions without a common measure, such a column of functions is obtained by the well-known process of seeking for the greatest common measure, reversing at each step the sign of the remainder: viz. we thus derive a set of functions $R, S, T, \ldots$ where

$$
\begin{aligned}
& P=\lambda Q-R, \\
& Q=\mu R-S, \\
& R=\nu S-T, \\
& S=\rho T-U, \\
& \vdots
\end{aligned}
$$

the degrees of the successive functions $R, S, T, \ldots$, being successively less and less, so that the last of them, say $V$, is an absolute constant: or we may stop the process as soon as we arrive at a function $V$, the sign of which remains unaltered for all values between the initial and final values of the variable. It may be observed that the process may be regarded as applicable in the case where the degree of $Q$ exceeds that of $P$ : viz. we then have $\lambda=0, R=-P$, and the column begins $(P, Q,-P, S, \ldots)$, the subsequent terms being, except as to sign, the same as if $P, Q$ had been interchanged.

Reversing the sign of $P$ or $Q$, we reverse in the bicolumn a set of alternate rows, and thus reverse the gain: and reversing both signs we reverse all the rows, 
and leave the gain unaltered-of course the intercalation (considered irrespectively of sign) is in each case unaltered. It is convenient to take the signs in such manner that for the initial value of $x$, the signs of $P, Q$ shall be each positive: or, what is the same thing, taking $P, Q$ with their proper signs, we may in the bicolumn, by reversing if necessary each or either set of alternate rows, make the left-hand column to begin with the signs ++ .

26. The complete rule now is-for a given trajectory form the bicolumn for $P Q R S . .$. , and if necessary, by reversing each or either set of alternate rows, make the left-hand column to begin with ++ : then if there is a gain the intercalation begins with $P$, if a loss with $Q$, the gain or loss showing the number of $P$ 's. To find the number of $Q$ 's prefix at the top of the bicolumn the second row reversed-then the gain or loss (equal to or differing by unity from the original value) shows the number of $Q$ 's. It may happen that for $P$ the gain is $=0$; then for $Q$ the gain is 0 or \pm 1 , and the intercalation vanishes or is $Q$.

27. I give some simple examples.

$$
\begin{array}{llllll} 
& & 0 & 2 & 4 \\
\cline { 3 - 5 } P=x-1 & - & + & + \\
Q= & x-3 & - & - & + \\
R= & -1 & - & - & - \\
\hline 0 & P & 2 & Q & 4
\end{array}
$$

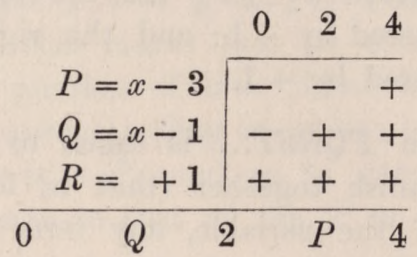

In the left-hand example taking the intervals to be successively $0-2,0-4,2-4$, the bicolumns modified as above are

viz.

\begin{tabular}{ll|llll}
$0-2$ & 0 & -4 & 2 & -4 \\
\hline- & - & - & + & - & + \\
\hline+ & - & + & - & + & + \\
+ & + & + & - & + & - \\
+ & + & + & + & - & -
\end{tabular}

Interval $0-2$; for $P$ gain $=1, P$ first; for $Q$ gain $=0$; Intercalation is $P$;

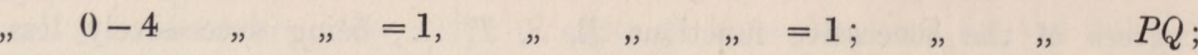

$$
\begin{aligned}
& " 2-4 \quad " \quad, \quad=0 \quad \ldots . . \quad, \quad \operatorname{loss}=1 ; \quad \# \quad, \quad Q .
\end{aligned}
$$

And similarly in the right-hand example we have

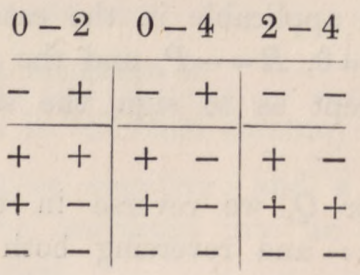


Interval $0-2$ for $P$ gain $=0$, for $Q$ gain $=-1$; Intercalation is $Q$;

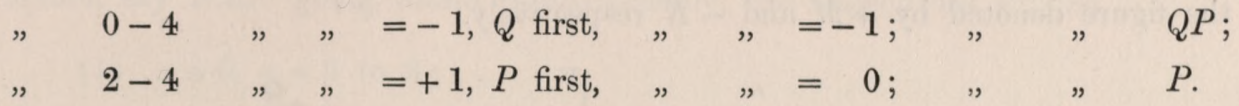

28. Or to take a slightly more complicated example,

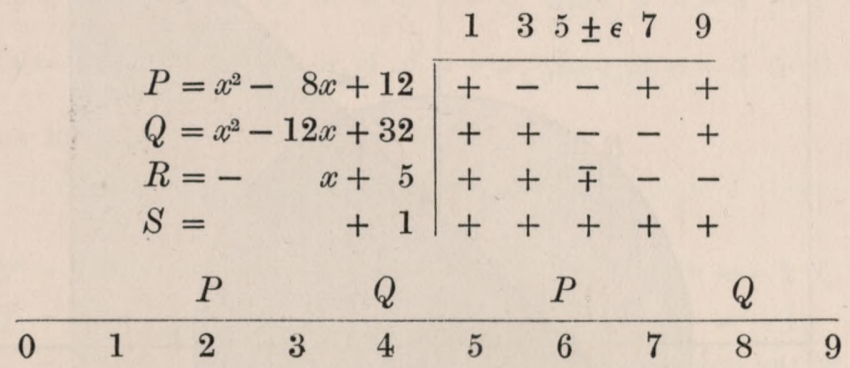

And hence for the several intervals,

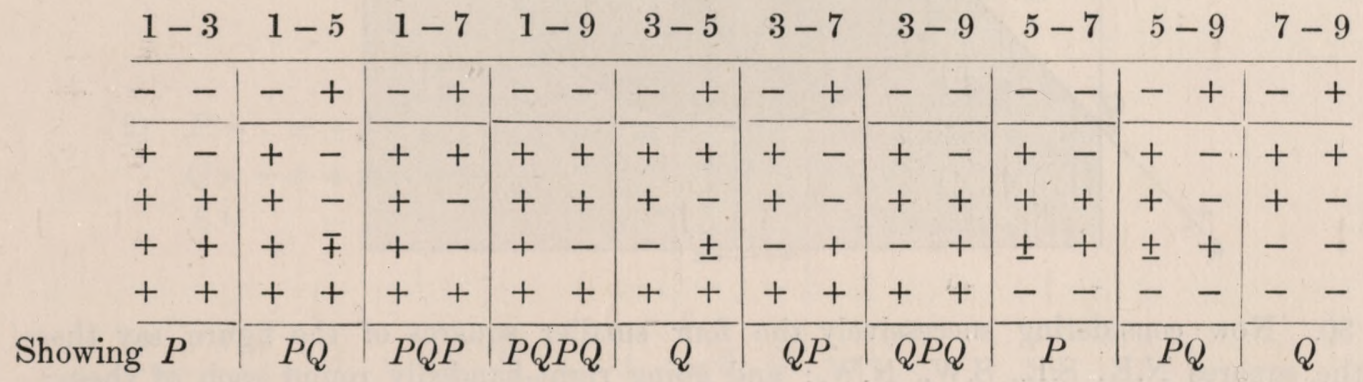

For instance :-

Interval $1-9$ for $P$ gain $=2, P$ first, for $Q$ gain $=2$ : Intercalation is $P Q P Q$.

It may be added that $P$ being + for $x=1$, the \pm intercalation is $+P Q P Q$.

29. As an example of circuits take the following: curves are $P=0, Q=0$, where

$$
\begin{aligned}
& P=x^{2}+y^{2}-4, \\
& Q=y-x-1
\end{aligned}
$$

viz. $P=0$ (see figure) is a circle, centre the origin, radius $=2:$ the inside hereof $(P=-)$ being coloured red: and $Q=0$ is a right line cutting the axes of $x, y$ at the points $(-1,0)$ and $(0,1)$ respectively, or say running N.E. and S.W., the lower region $(Q=-)$ being coloured blue: the square is an arbitrary circuit $(x= \pm 3, y= \pm 3)$ surrounding the circle, and the regions within the square are coloured by what precedes sable, gules, azure, argent, as shown in the figure: the line and circle intersect in two points $M, N$. Going right-handedly round these respectively, for $M$ the order is sable, gules, argent, azure, viz. $M$ is a right-handed root; while for $N$ the order is 
sable, azure, argent, gules, viz. $N$ is a left-handed root: the two points are accordingly in the figure denoted by $+M$ and $-N$ respectively.

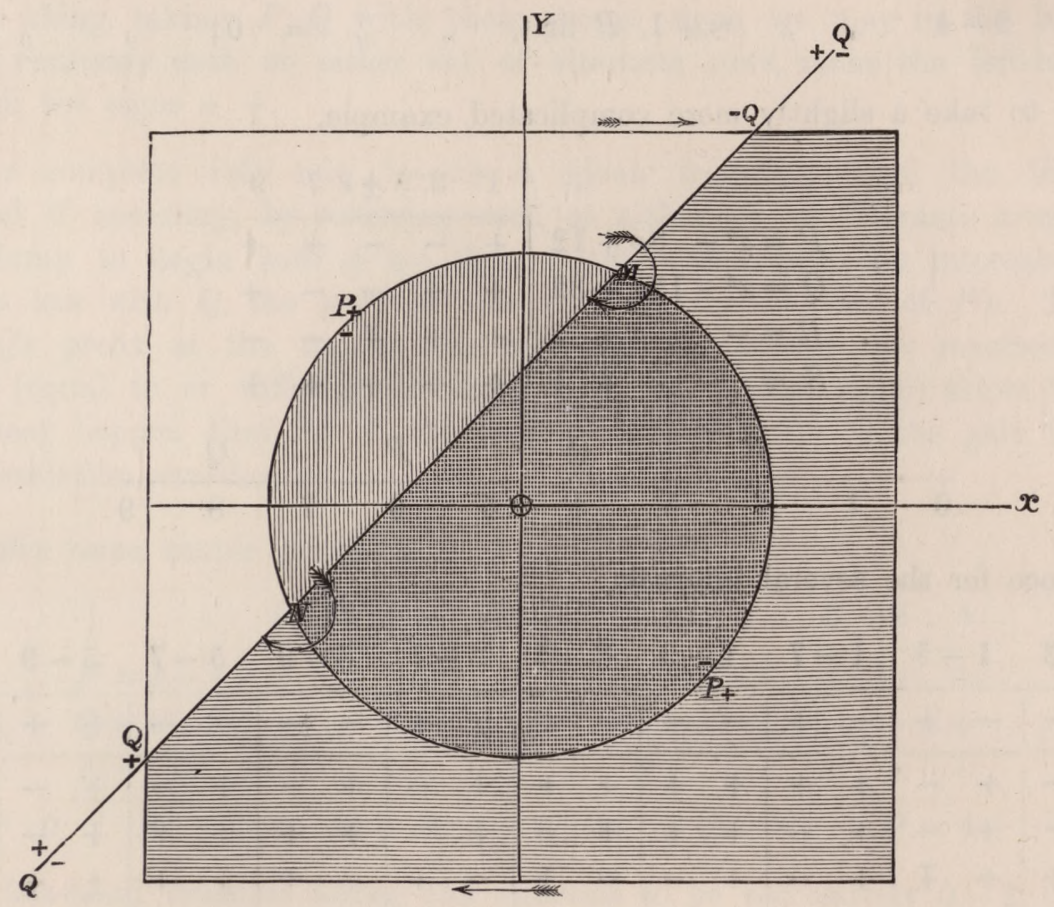

30. Now considering successively the four smaller squares of the figure, say these are the squares N.E., S.E., S.W., N.W.: and going right-handedly round each of these:

In the square N.E., the sequence and therefore also the intercalation is $+P-Q+P-Q$, viz. this is an intercalation $(+P-Q)$, showing an excess 1 of right-handed roots, and of course consisting with the single right-handed root $M$.

In the square S.E., the sequence is $-P+P$, viz. this is an intercalation $(P Q)_{0}$, showing an equality of right- and left-handed roots, and consisting with no root.

In the square S.W., the sequence and therefore also the intercalation is $-P+Q-P+Q$ : viz. this is an intercalation $(-P+Q)_{1}$, showing an excess 1 of left-handed roots, and consisting with the single left-handed root $N$.

And in the square N.W., the sequence is $-Q+P-P+Q$, viz. this is an intercalation $(P Q)_{0}$, showing an equality of right- and left-handed roots, and consisting with no root.

Again take the whole large square: the sequence is $-Q+Q$ : viz. the intercalation is $(P Q)_{0}$, showing an equality of right- and left-handed roots, and consisting with there being one of each.

So taking the squares N.E. and N.W. conjointly, the sequence and therefore also the intercalation is $-Q+P-Q+P$, viz. this is an intercalation $(+P-Q)_{1}$, as for the single square N.E. 
31. As regards the analytical determination it will be sufficient to consider a single square, say N.E.: going round right-handedly, the trajectories will be

(1) $x=0, y=0$ to 3 ;

(2) $y=3, x=0$ to 3 ;

(3) $x=3, y=3$ to 0 ; or if $y^{\prime}=-y$, then $y^{\prime}=-3$ to 0 ;

(4) $y=0, x=3$ to 0 ; or if $x^{\prime}=-x$, then $x^{\prime}=-3$ to 0 .

And we thus have

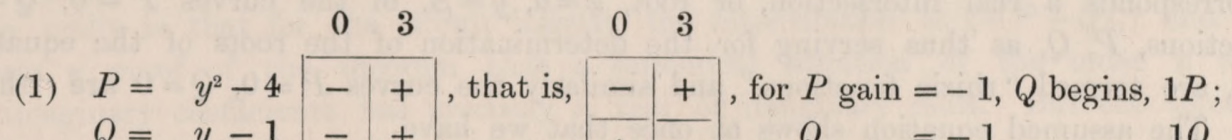

$Q=y-1-+\quad+-\overline{-}, \quad Q=-1, \quad \# \quad 1 Q$.

\begin{tabular}{ll|l}
$R=-1+|-|$ & $\begin{array}{l}+ \\
+ \\
-\end{array}-$ Intercalation is $Q P$, or since at \\
- & origin $P=-, R=-$, or region
\end{tabular}

is sable, it is

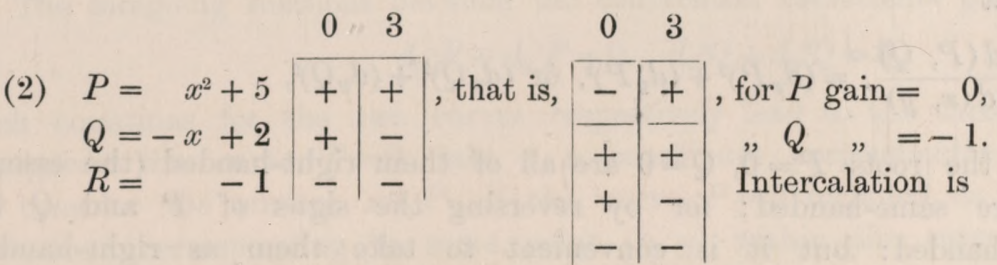

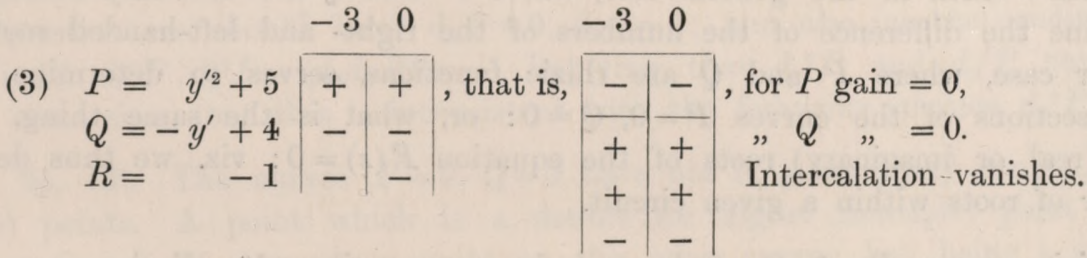

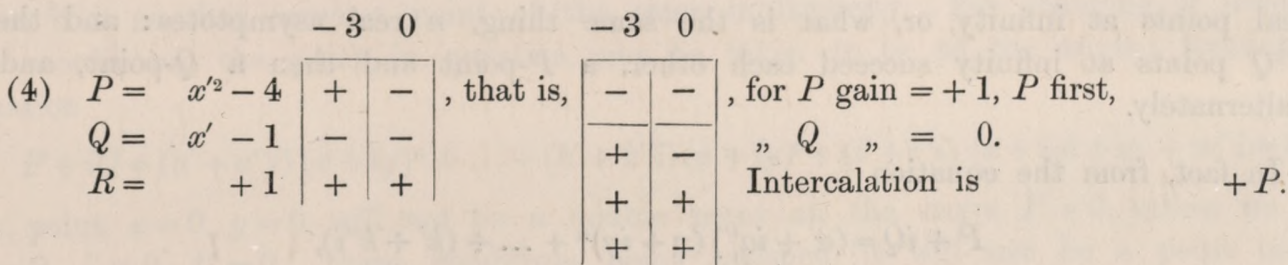

Hence for the four sides, combining the intercalations, we have $-Q+P-Q+P$, and since there are no terms to be omitted, this is the intercalation of the N.E. square: which is right.

C. IX. 


\section{The Rhizic Theory. Articles Nos. 32 to 38.}

32. Consider now $F(z)=(*)(z, 1)^{n}$ a rational and integral function of $z$, of the order $n$ with in general imaginary (complex) coefficients, or, what is the same thing, let $F^{\prime}(z)=f(z)+i \phi(z)$, where the functions $f, \phi$ are real*. Writing herein $z=x+i y$, let $P, Q$ be the real part and the coefficient of the imaginary part in the function $F(x+i y)$ : or, what is the same thing, assume

$$
P+i Q=f(x+i y)+i \phi(x+i y),
$$

then it is clear that to any root $\alpha+i \beta$ (real or imaginary) of the equation $F(z)=0$, there corresponds a real intersection, or root, $x=\alpha, y=\beta$, of the curves $P=0, Q=0$. The functions, $P, Q$, as thus serving for the determination of the roots of the equation $F(z)=0$, are termed "rhizic functions," and similarly the curves $P=0, Q=0$ are "rhizic curves." The assumed equation shows at once that we have

or, what is the same thing,

$$
d_{y}(P+i Q)=i d_{x}(P+i Q)
$$

And we hence see that

$$
d_{y} P=-d_{x} Q, d_{x} P=d_{y} Q
$$

$$
\frac{d(P, Q)}{d(x, y)},=\left(d_{x} P\right)^{2}+\left(d_{y} P\right)^{2}, \text { or }\left(d_{x} Q\right)^{2}+\left(d_{y} Q\right)^{2},
$$

is positive: viz. that the roots $P=0, Q=0$ are all of them right-handed (the essential thing is that they are same-handed; for by reversing the signs of $P$ and $Q$ they might be made left-handed: but it is convenient to take them as right-handed): hence the theorem-which in the general case, where $P$ and $Q$ are arbitrary functions, serves to determine the difference of the numbers of the right- and left-handed rootsin the particular case, where $P$ and $Q$ are rhizic functions, serves to determine the number of intersections of the curves $P=0, Q=0$ : or, what is the same thing, the number of the (real or imaginary) roots of the equation $F^{\prime}(z)=0$ : viz. we thus determine the number of roots within a given circuit.

33. The rhizic curves $P=0, Q=0$ have various properties. $1^{\circ}$. Each curve has $n$ real points at infinity, or, what is the same thing, $n$ real asymptotes: and the $P$ and $Q$ points at infinity succeed each other, a $P$-point and then a $Q$-point, and so on, alternately.

In fact, from the equation

$$
P+i Q=\left(a^{\prime}+i a^{\prime \prime}\right)(x+i y)^{n}+\ldots+\left(k^{\prime}+k^{\prime \prime} i\right),
$$

writing herein $a^{\prime}+i a^{\prime \prime}=a(\cos \alpha+i \sin \alpha)$, and $x+i y=\rho(\cos \theta+i \sin \theta)$, we have

$$
P+i Q=a \rho^{n}[\cos (n \theta+\alpha)+i \sin (n \theta+\alpha)]+\ldots+k^{\prime}+k^{\prime \prime} i .
$$

* It is assumed that the equation $F(z)=0$ has no equal roots: this being so, the curves $P=0, Q=0$, will have no point of multiple intersection; which accords with the assumption made in the general case of two arbitrary curves. 
It thus appears that for the curve $P=0$, the points at infinity are given by the equation $\cos (n \theta+\alpha)=0$; while for the curve $Q=0$, they are given by the equation $\sin (n \theta+\alpha)=0$ : which proves the theorem.

Representing infinity as a closed curve or circuit, each point at infinity must be represented by two opposite points on the circuit; so that writing down $P$ for each $P$-point and $Q$ for each $Q$-point, we have $2 n P$ 's and $2 n Q$ 's succeeding each other, a $P$-point and then a $Q$-point, and so on, alternately.

It may be assumed that taking the circuit right-handedly, the $P$ 's are + and the Q's -, (this depends only on the colouring, but it corresponds with the foregoing assumption that the roots $P=0, Q=0$ are right-handed): the theorem just obtained then really is that for the circuit infinity, the intercalation is $(+P-Q)_{n}$ : and we have herein a proof of the theorem that a numerical equation of the order $n$ with real or imaginary coefficients has precisely $n$ real or imaginary roots. But the force of this will more distinctly appear presently.

34. $2^{\circ}$. Neither of the curves $P=0, Q=0$ can include as part of itself a closed curve or circuit.

The foregoing relations between the differential coefficients give

$$
d_{x}{ }^{2} P+d_{y}{ }^{2} P=0, \quad d_{x}{ }^{2} Q+d_{y}{ }^{2} Q=0,
$$

which equations for the two curves respectively lead to the theorem in question. For as regards the curve $P=0$, take $z$ a coordinate perpendicular to the plane of $x y$, and consider the surface $z=P$ : if the curve $P=0$ included as part of itself a closed curve, then corresponding to some point $(x, y)$ within the curve we should have $z$ a proper maximum or minimum, viz. there would be a summit or an imit; at the point in question we should have $d_{x} P=0, d_{x} Q=0$; and also (as the condition of a summit or imit) $d_{x}{ }^{2} P \cdot d_{y}{ }^{2} P-\left(d_{x} d_{y} P\right)^{2}=+$, implying that $d_{x}{ }^{2} P$ and $d_{y}{ }^{2} P$ have at this point the same sign: but this is inconsistent with the foregoing relation $d_{x}{ }^{2} P+d_{y}{ }^{2} P=0$.

35. 3 . The curves $P=0, Q=0$ have not in general any double (or higher multiple) points. A point which is a double (or higher multiple) point on one of these curves is not of necessity a point on the other curve: but being a point on the other curve it is on that curve a point of the same multiplicity. For changing if necessary the coordinates, the point in question may be taken to be at the origin: forming the equation

$$
P+i Q=\left(a^{\prime}+a^{\prime \prime} i\right)(x+i y)^{n}+\ldots+\left(k^{\prime}+k^{\prime \prime} i\right)(x+i y)^{2}+\left(l^{\prime}+l^{\prime \prime} i\right)(x+i y)+m^{\prime}+m^{\prime \prime} i=0,
$$

the point $x=0, y=0$ will not be a double point on the curve $P=0$, unless we have $m^{\prime}=0, \quad l^{\prime}=0, \quad l^{\prime \prime}=0$; these conditions being satisfied, it will not be a point on the curve $Q=0$ unless also $m^{\prime \prime}=0$; but this being so, it will be a double point on the curve $Q=0$ : and the like for points of higher multiplicity. But a point which is a multiple point on each curve, represents four or more coincident intersections of the curves $P=0, Q=0$, that is, four or more equal roots of the equation $F^{\prime}(z)=0$; so that assuming that the equation has no equal roots, the case does not arise: and we in fact exclude it from consideration. 
To fix the ideas assume that the curves $P=0, Q=0$ are each of them without double points. As already seen, neither of them includes as part of itself a closed curve. Hence in the figure the curve $P=0$ must consist of $n$ branches each drawn from a point $P$ in the circuit (viz. the circuit infinity) to another point $P$ in the circuit; and in such manner that no two branches intersect each other: this implies that the two points $P$ of the same branch must include between them an even number (which may of course be $=0$ ) of points $P$. And the like as regards the curve $Q=0$.

36. $4^{\circ}$. No branch of the $P$-curve can meet a branch of the $Q$-curve more than once. In fact, drawing the two branches to meet twice, the colouring would at once show that of the two intersections or roots, one must be right, the other left-handed: whence, the roots being all right-handed, the branches do not meet twice. And in exactly the same way it appears that no $P$-branch can meet two $Q$-branches, or any $Q$-branch meet two $P$-branches. And under these restrictions it requires only a consideration of a few successive cases to show that the $n P$-branches, and the $n Q$-branches can only be drawn on the condition that each $P$-branch shall intersect once and only once a single $Q$-branch; which of course implies that each $Q$-branch intersects once and once only a single $P$-branch: and further, that there shall be precisely $n$ intersections: viz. the $n P$-branches and the $n Q$-branches must satisfy the conditions just stated. And the theorem of the $n$ roots is thus obtained as a consequence of the impossibility (except under the same conditions) of drawing the $n P$-branches and the $n Q$-branches, so as to give rise to right-handed roots only. But the case of double or higher multiple points would need to be specially considered.

37. It is interesting for a given value of $n$ to consider $\phi(n)$, the number of different ways in which the $P$-branches and the $Q$-branches can be drawn. We have $2 n$ points $P$ and $2 n$ points $Q$, in all $4 n$ points : starting from any point $P$, these may be numbered in order $1,2,3, \ldots, 4 n$, the points $P$ bearing odd numbers and the points $Q$ even numbers. We may consider the $P$-branch which joins 1 with some $P$-point $\beta$, and (intersecting this) the $Q$-branch which joins some two $Q$-points $\alpha$ and $\gamma$ : the numbers $1 \alpha \beta \gamma$ are then in order of increasing magnitude: and excluding these four points there remain the points corresponding to numbers between 1 and $\alpha$, between $\alpha$ and $\beta$, between $\beta$ and $\gamma$, and between $\gamma$ and 1. Now since the $P$-branch $1 \beta$ meets the $Q$-branch $\alpha \gamma$, no branch from a point between 1 and $\alpha$ can meet either of these curves; hence these points form a system by themselves, capable of being connected together by $P$-branches and $Q$-branches: the number of them must therefore be a multiple of 4 : and the like as to the points between $\alpha$ and $\beta$, between $\beta$ and $\gamma$, and between $\gamma$ and 1. Taking the number of the points in the four systems to be $4 x, 4 y, 4 z$, and $4 w$ respectively, we have $x+y+z+w=n-1$, and the first-mentioned four points bear the numbers

$$
\begin{gathered}
1, \\
\alpha=4 x+2, \\
\beta=4 x+4 y+3 \\
\gamma=4 x+4 y+4 z+4
\end{gathered}
$$


For the four systems the number of ways of drawing the $P$ - and $Q$-branches are $\phi x, \phi y, \phi z, \phi w$ respectively: that is, $x, y, z, w$ being any partition whatever of $n-1$ (order attended to), and $\phi(0)$ being $=1$, we have

$$
\phi(n)=\Sigma \phi(x) \phi(y) \phi(z) \phi(w),
$$

which is the condition for the determination of $\phi n$.

Taking then $\theta$ for the value of the generating function

$$
1+t \phi(1)+t^{2} \phi(2) \ldots+t^{n} \phi(n)+\ldots,
$$

it hereby appears that we have

$$
\theta=1+t \theta^{4}
$$

or writing this for a moment $\theta=u+t \theta^{4}$, and expanding by Lagrange's theorem, but putting finally $u=1$, we have the value of $\theta$, that is of the generating function,

that is,

$$
\begin{aligned}
& =1+[4]^{0} \frac{t}{1}+[8]^{1} \frac{t^{2}}{1.2}+[12]^{2} \frac{t^{3}}{1.2 .3} \ldots \ldots+[4 n]^{n-1} \frac{t^{n}}{1.2 \ldots n}+\ldots \\
& =1+t+4 t^{2}+22 t^{3}+140 t^{4}+\ldots,
\end{aligned}
$$

and generally

$$
\phi(1)=1, \quad \phi(2)=4, \quad \phi(3)=22, \quad \phi(4)=140, \ldots
$$

$$
\phi(n)=\frac{[4 n]^{n-1}}{[n]^{n}}, \quad=\frac{4 n \cdot 4 n-1 \ldots 3 n+2}{2.3 \ldots n} .
$$

The results are easily verified for the successive particular cases; thus $n=1$, the points are $1,2,3,4$, and the $P$ - and $Q$-branches respectively are 13, 24: $\phi(1)=1$. Again $n=2$, the points are $1,2,3,4,5,6,7,8$ : we may join 13,24 or 13, 28 or 17, 28 or 17, 68, leaving in each case four contiguous numbers which may be joined in a single manner: that is, $\phi(2)=4$. Or, what is the same thing, the partitions of 1 are $0001,0010,0100,1000$, whence $\phi(2)=4\{\phi(0)\}^{3} \phi(1)=4$. Again $n=3$, the partitions of 2 are 0002 , \&c. ( 4 of this form) and 1100 ( 6 of this form): that is, $\phi(3)=4\{\phi(0)\}^{3} \phi(2)+6\{\phi(0)\}^{2}\{\phi(1)\}^{2},=4.4+6.1=22$, and so on.

38. Starting from the $4 n$ points $P$ and $Q$, and joining them in any manner subject to the foregoing conditions, we have a diagram representing two rhizic curves; and colouring the regions we verify that the $n$ roots are all of them right-handed. We have for instance the annexed figure $(n=3)$.

Having drawn such a figure we may, by a continuous variation of the several lines, in a variety of ways introduce a double point in the $P$-curve, or in the $Q$-curve: and by a continued repetition of the process introduce double points in each or either curve: thus for instance we may from the last figure derive a new figure in which the $P$-curve has a node at $N$. It will be observed that here it is no longer the case that each $P$-branch intersects one and only one $Q$-branch: the $P$-branch $1-9$ does not meet any $Q$-branch, but the $P$-branch $7-11$ meets two $Q$-branches. But looking at the figure in a different manner, and considering the $P$-branches through $N$ as 
being either $11-N-1$ and $7-N-9$, or $1-N-7$ and $9-N-11$, then in either case each $P$-branch intersects one and only one $Q$-branch: and in this way, in a

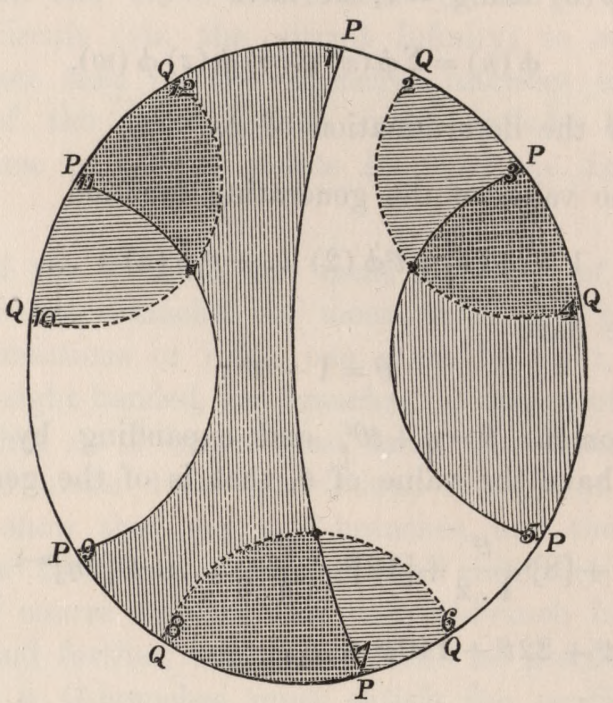

diagram in which the two curves have each or either of them double points, but neither curve passes through a double point of the other curve, the theorem may be regarded as remaining true-we in fact consider the diagram as the limit of a diagram wherein the curves have no double points. It will be recollected that, the equation

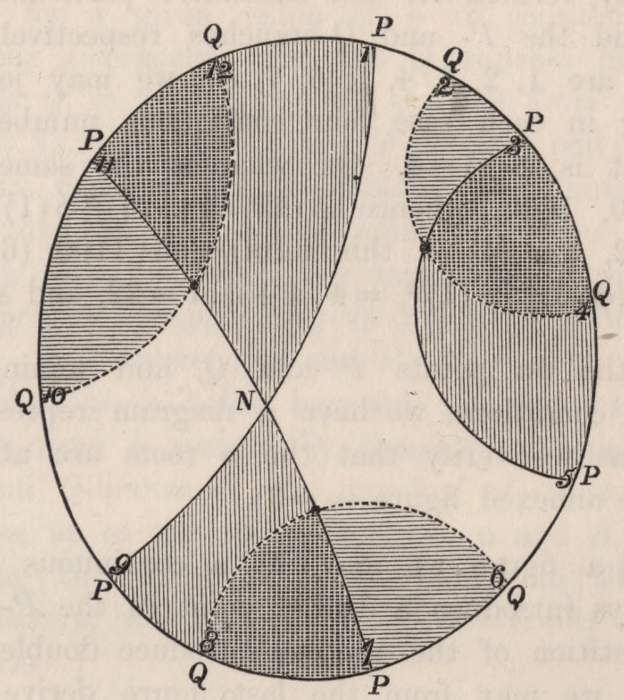

$F(z)$ being without equal roots, we cannot have either curve passing through a multiple point of the other curve. And we thus see that the various figures drawn as above without double points are, so to speak, the types of all the different forms of a system of rhizic curves $P=0, Q=0$. 
In connexion with the present paper I give the following list of Memoirs :-

Cauchy. Calcul des Indices des fonctions. Jour. de l'ÉEcole Polyt. t. xv. (1837), pp. 176-229. First part seems to have been written in 1833: second part is dated 20th June, 1837. Refers to a memoir presented to the Academy of Turin the 17th Nov. 1831, wherein the principles of the "Calcul des Indices des fonctions" are deduced from the theory of definite integrals: I have not seen this.

Sturm and Liouville. Démonstration d'un théorème de M. Cauchy relative aux racines imaginaires des équations. Liouv. t. I. (1836), pp. 278-289.

Sturm. Autres démonstrations du même théorème. Liouv. t. I. (1836), pp. 290-308.

These two papers contain proofs of the particular theorem relating to the roots of an equation $F^{\prime}(z)=0$, but do not refer to the general theorem relating to the intersection of the two curves $P=0, Q=0$ : the special theorem of the existence of the $n$ roots of the equation $F(z)=0$ is considered.

Sylvester. A theory of the syzygetic relations of two rational integral functions, comprising an application to the theory of Sturm's functions and that of the greatest algebraical common measure. Phil. Trans. t. cxlIII. (1853), pp. 407-548.

De Morgan. A proof of the existence of a root in every algebraic equation, with an examination and extension of Cauchy's theorem on imaginary roots, and remarks on the proofs of the existence of roots given by Argand and Mourey. Camb. Phil. Trans. t. x. (1858), pp. 261-270.

Contains the important remark that the two curves $P=0, Q=0$ are such that two branches, one of each curve, cannot inclose a space; also that the two curves always [i.e. at a simple intersection] intersect orthogonally.

Airy, G. B. Suggestion of a proof of the theorem that every algebraic equation has a root. Camb. Phil. Trans. t. x. (1859), pp. 283-28.9.

Cayley, A. Sketch of a proof of the theorem that every algebraic equation has a root. Phil. Mag. t. xviII. (1859), [248], pp. 436-439.

walton, w. On a theorem in maxima and minima. Quart. Math. Jour. t. x. (1870), pp. 253-262. Cayley, A. Addition thereto, [562], pp. 262, 263. (Relates to the curves $P=0, Q=0$.)

Walton, w. Note on rhizic curves. Quart. Math. Jour. t. XI. (1871), pp. 91-98. First use of the term "rhizic curves:" relates chiefly to the configuration of each curve at a multiple point, and of the two at a common multiple point.

walton, w. On the spoke-asymptotes of rhizic curves. Quart. Math. Jour. t. XI. (1871), pp. 200-202.

walton, w. On a property of the curvature of rhizic curves at multiple points. Quart. Math. Jour. t. XI. (1871), pp. 274-281.

Björling. Sur la séparation des racines d'équations algébriques. Upsala, Nova Acta Soc. Sci. (1870), pp. 1-35. (Contains delineations of some rhizic curves.) 